\title{
Valoración de los patrones de consumo alimentario y actividad física en universitarios de tres carreras respecto a las guías alimentarias para la población argentina
}

\section{Assessment of dietary consumption patterns and physical activity of students from three university degree programs with respect to dietary guidelines for the Argentine population}

\section{RESUMEN}

Numerosos estudios coinciden en que el inicio de la vida universitaria se asocia a cambios poco saludables en prácticas alimentarias y estilos de vida que pueden consolidarse en la edad adulta. El objetivo fue valorar en qué medida estudiantes de tres carreras cumplen con las recomendaciones de las nuevas Guías Alimentarias para la Población Argentina de 2016. Se encuestaron 164 alumnos de Bioquímica (BQ), Licenciatura en Nutrición (LN) y Licenciatura en Biotecnología (LB) de la Universidad Nacional del Litoral. Los alumnos de $L N$ se acercaron más a las recomendaciones que $B Q$ y LB: mayor porcentaje realizaba cuatro comidas $(45,0 \%$ vs $22,4 \% L B$ y $21,8 \% B Q, p=0,009)$ y consumía diariamente leche/yogur ( $p=0,042)$; los estudiantes de BQ presentaron el menor consumo de frutas $(p=0,000)$, mientras los de $L B$ presentaron la menor ingesta de verduras $(p=0,023)$. El $79,3 \%$ del total realizaba actividad física y de ellos 59,8\% alcanzó los 30 minutos diarios recomendados. La Universidad ofrece espacios de alimentación saludable (comedor universitario) y actividad física (predio de deportes) que no resultaban suficientemente aprovechados. Se concluye que resulta necesario planificar intervenciones para fomentar y/o mejorar patrones, consumo de alimentos y actividad física acordes a estilos más saludables en estos universitarios. Palabras clave: Actividad física; Consumo de alimentos; Guías Alimentarias para la Población Argentina; Patrón de ingesta; Universitarios.

\footnotetext{
ABSTRACT

Numerous studies agree that the beginning of university life is associated with unhealthy changes in eating practices and lifestyles that can be consolidated in adulthood. The aim of this study was to assess the extent to which the nutrition and physical activity of university students from three career programs comply with the recommendations of the new Dietary Guidelines for the Argentine Population (2016). One hundred and sixty-four (164) students of Biochemistry (BQ), Bachelor of Nutrition (LN) and Bachelor of Biotechnology $(L B)$ of the Universidad Nacional del Litoral were surveyed. LN students approached the recommendations more than BQ and LB students: a higher percentage had
}

\author{
Alejandra Fortino ${ }^{1 *}$, Matías Vargas ${ }^{1}$, Eugenia Berta ${ }^{2}$,
} Florencia Cuneo ${ }^{3}$, Olga Ávila².

1. Laboratorio de Estudio de Enfermedades Metabólicas Relacionadas con la Nutrición. Facultad de Bioquímica y Ciencias Biológicas, Universidad Nacional del Litoral. Santa Fe, Argentina.

2. Departamento de Matemática. Facultad de Bioquímica y Ciencias Biológicas, Universidad Nacional del Litoral. Santa Fe, Argentina. 3. Cátedra de Seminario y Trabajo Final. Facultad de Bioquímica y Ciencias Biológicas, Universidad Nacional del Litoral. Santa Fe, Argentina.

* Dirigir correspondencia: María Alejandra Fortino. Facultad de Bioquímica y Ciencias Biológicas, Universidad Nacional del Litoral. Ciudad Universitaria, RN 168 Km 0. (S3000) Santa Fe, Argentina. Email afortino@fbcb.unl.edu.ar

Este trabajo fue recibido el 13 de febrero de 2020. Aceptado con modificaciones: 11 de junio de 2020. Aceptado para ser publicado: 21 de julio de 2020.

four meals per day (45.0\% LN vs. $22.4 \%$ LB and $21.8 \%$ $B Q, p$-value $=0.009)$ and consumed a daily portion of milk/ yogurt ( $p$-value $=0.042)$; the BQ students had the lowest fruit consumption ( $p$-value $=0.000)$, while the $L B$ students had the lowest vegetable intake ( $p$-value $=0.023) . A / I$ students presented patterns of intake and consumption of foods strongly influenced by the Argentine culture. Almost eighty percent (79.3\%) of students did physical activity, but only $59.8 \%$ reached the recommended 30 minutes a day. The University offers healthy eating spaces (university canteen) and physical activity (sports grounds) that were not sufficiently exploited. It is concluded that it is necessary to plan interventions to promote and / or improve patterns of food consumption and physical activity, according to 
healthier lifestyles for university students.

Keywords: university students; intake patterns; food consumption; physical activity; Dietary Guidelines for the Argentine Population (GAPA).

\section{INTRODUCCIÓN}

En 2004, la Organización Mundial de la Salud (OMS) ${ }^{1}$ propuso la estrategia "Régimen Alimentario, Actividad Física y Salud" alentando a los países a formular y actualizar directrices en relación a la prevención de factores de riesgo de enfermedades crónicas no transmisibles (ECNT), en función de los hábitos y cultura propios de los países. Sin embargo, recientemente la OMS ha publicado un último informe reconociendo la falta de progresos de la estrategia a nivel global. Las ECNT, representadas principalmente por enfermedades cardiovasculares, diabetes, obesidad, cáncer y enfermedades respiratorias crónicas, se asocian a factores de riesgo (FR) como la alimentación inadecuada, la inactividad física, el consumo de tabaco y el alcohol ${ }^{2}$. Datos en Argentina muestran a las enfermedades cardiovasculares como primera causa de muerte $(40 \%)$, registrando un incremento del 5,5\% entre 2015 y $2016^{3}$. Estudios previos en estudiantes de la Universidad Nacional del Litoral (Santa Fe, Argentina), indican que los FR de ECNT se hallan presentes aún en carreras relacionadas a la salud. Investigaciones previas demostraron que el $75,7 \%$ de los estudiantes de nutrición poseían FR familiares y/o personales; destacando entre los últimos el consumo de alcohol, la inactividad física y la hipercolesterolemia (con disminución de HDL-col y elevación de LDL-col) ${ }^{4}$, mientras que en estudiantes de medicina la dieta inadecuada fue el FR de mayor prevalencia, seguida por el exceso de grasa corporal, la baja actividad física, el índice de masa corporal elevado y el riesgo cardiovascular asociado a la circunferencia abdominal ${ }^{5}$. Por tanto, los estudiantes de la UNL no escapan al impacto de los FR demostrados a nivel internacional en estudiantes de la salud ${ }^{6}$.

A partir de la "Carta de Ottawa"7, internacionalmente se considera la universidad como un ámbito especialmente propicio para la promoción de hábitos saludables. Para los estudiantes, la universidad es la última instancia de educación formal que puede contribuir a la adquisición de estilos de vida saludables. El período universitario merece especial atención, ya que los estudiantes transitan por un proceso complejo de cambios: aumento de la autonomía, elevada carga horaria de estudio, alto grado de estrés y ansiedad, y en ocasiones abandono del domicilio familiar. Todo ello puede influir en cambios en los patrones de alimentación, horarios de comidas o compra de alimentos. Numerosos trabajos han mostrado que estos cambios no siempre están asociados a prácticas saludables, dado que es un colectivo que tiende a conductas tales como disminución de la actividad física, omisión de comidas, picoteo entre horas, aumento del consumo de comidas rápidas, dietas extremas de adelgazamiento, ingesta de nutrientes desequilibrada, consumo en exceso bebidas alcohólicas y/o azucaradas, entre otras ${ }^{8,9,10}$. La dieta de los jóvenes universitarios plantea un importante reto para la Educación Alimentaria Nutricional (EAN), ya que implica la consolidación de patrones alimentarios que en la edad adulta pueden contribuir favorable o desfavorablemente al riesgo de ECNT. Así lo reconoce Argentina en el "Manual de Universidades Saludables"11, instando a la generación de líneas de investigación y de acción que permitan avanzar en el desarrollo de estrategias educativas y comunitarias, así como reorientar programas para promover la salud.

Como parte del sistema de vigilancia y prevención de ECNT, el Ministerio de Salud de la Nación (MSAL) revisó en 2016 las recomendaciones alimentarias saludables para la población general, tal como sugieren los organismos internacionales, y se publicaron las Guías Alimentarias para la Población Argentina (GAPA 2016) ${ }^{12}$. Las mismas pueden resumirse en 10 mensajes principales y una Gráfica de Alimentación Diaria (plato alimentario) que los representa. Las recomendaciones de consumo se basan en grupos de alimentos y son consideradas un instrumento útil para realizar EAN promoviendo la adopción de estilos de vida saludables.

El presente trabajo forma parte de una investigación en estudiantes de la Facultad de Bioquímica y Ciencias Biológicas de la Universidad Nacional del Litoral (FBCBUNL), Santa Fe, Argentina. Los objetivos del mismo fueron valorar en qué medida la alimentación y la actividad física de los universitarios de tres carreras cumplen con las recomendaciones de una alimentación saludable (GAPA 2016) y determinar posibles diferencias entre las mismas.

\section{MATERIALES Y MÉTODOS}

Se realizó un estudio transversal, descriptivo y comparativo, durante mayo-julio de 2017, mediante una encuesta a 164 alumnos de las carreras de Bioquímica (BQ, $\mathrm{n}=55$ ), Licenciatura en Nutrición ( $\mathrm{LN}, \mathrm{n}=60$ ) y Licenciatura en Biotecnología ( $L B, n=49)$. El procedimiento que se detalla a continuación para la recolección de datos, así como la propia encuesta, contó con el aval y la aprobación del Comité Asesor de Ética y Seguridad de la Investigación (CAESI) de la FBCB-UNL. Se utilizó una técnica de muestreo no probabilístico consecutivo, para la aplicación de una encuesta autoadministrada que exigió como criterio de inclusión que los estudiantes estuviesen cursando el segundo año (según los programas curriculares de cada carrera). La condición estipulada como criterio de inclusión obedeció al cumplimiento de dos realidades: contar con experiencia en la vida universitaria y encontrarse en una etapa inicial de la misma. La representatividad de la muestra se calculó mediante fórmula para poblaciones finitas ${ }^{13}$.

A cada universitario se le explicó el propósito del estudio antes de participar, respondiendo voluntariamente y en forma anónima una encuesta semiestructurada en presencia de nutricionistas que los asesoraron ante cualquier duda. La encuesta, de elaboración propia, fue diseñada por expertos en nutrición para responder a los objetivos del 
estudio. La misma se validó previamente a su aplicación mediante prueba piloto y juicio de expertos en Nutrición (diferentes a los que intervinieron en su elaboración). Los aspectos juzgados en dicho instrumento para su validación fueron: validez de contenido, de constructo y de criterio; la confiabilidad medida por el coeficiente alfa de Cronbach fue de 0,89 .

La encuesta presentó las siguientes secciones:

1. Datos personales: edad, género, lugar de procedencia, año de ingreso universitario, horas de estudio presencial diario en la facultad, existencia de patologías que condicionen las selecciones alimentarias.

2. Actividad física: tipo, frecuencia y duración de cada sesión.

3. Patrones de consumo: realización de las comidas diarias (desayuno, almuerzo, merienda, cena), tiempo dedicado, lugar de realización y compañía.

4. Consumo de alimentos: tipo de alimento y frecuencia (diario y veces por semana); formas de preparación; agregado de sal a las comidas listas para el consumo.

5. Consumo de bebidas alcohólicas y no alcohólicas: tipo, frecuencia de consumo y versiones seleccionadas (con o sin azúcar).

El diseño de la sección 1 se orientó a la recolección de datos para la caracterización de la muestra (datos personales) y del contexto de su vida universitaria. Los datos de las secciones 2 a 5 se utilizaron para la valoración objeto de estudio, tomando como referencia las recomendaciones de las Guías Alimentarias para la Población Argentina (GAPA 2016) a partir de sus Mensajes y submensajes. En particular, la sección 4 (consumo de alimentos) se analizó según la clasificación por grupos de alimentos de dicho documento, a saber: carnes y huevos; leche, yogur y queso; verduras y frutas; feculentos cocidos (legumbres, cereales, vegetales feculentos y pan); aceites, frutas secas y semillas; y un grupo opcional: dulces y grasas. Si bien las GAPA no especifican el tiempo que debe dedicarse a cada comida, se consideró al menos 30 minutos para almuerzo y cena, y 15 minutos para desayuno o merienda ${ }^{14}$.

Para el análisis cuantitativo se utilizaron estadísticos descriptivos: media \pm desviación estándar (DE), frecuencias absolutas y porcentajes. Para establecer diferencias en variables entre las tres carreras se utilizó la prueba Chi cuadrado con software estadístico (Epidat 3.1), aceptando un nivel de significancia de 0,05. Las variables que mostraron significancia se destacan de forma expresa, de no mediar esta indicación la variable no presentó diferencia significativa entre carreras.

\section{RESULTADOS}

Caracterización general: la edad promedio fue 20,6 $6 \pm$ 2,5 años, con predominio femenino $(75,6 \%$ ) y un $16,1 \%$ refirió estar viviendo alejado de su seno familiar. Si bien los estudiantes se encontraban cursando asignaturas del segundo año del programa curricular de sus carreras, los resultados indicaron que se encontraban transitando su tercer año de vida universitaria, demostrando un desfase entre el currículo real y el programado. La carga de estudio presencial promedio fue de 5,48 h diarias. El 6,1\% manifestó presentar condicionamientos que podrían limitar el consumo de determinados alimentos por patologías (intolerancias, gastritis, entre otras, sin registrarse casos de celiaquía) o vegetarianismo. La tabla 1 muestra los resultados de las variables discriminadas por carrera.

Tabla 1. Características generales de la población discriminada por carreras. Los valores que no comparten superíndice son diferentes significativamente $(\mathrm{p}<0.05)$.
Característica
LN (n= 60)
BQ (n= 55)
LB $(n=49)$

Género (\%)

Femenino

Masculino

Edad promedio (años)

Años en la Universidad

Horas de estudio presencial (por día)

Lugar de procedencia (\%)

Ciudad de Santa Fe

Otros

Condiciones alimentarias (\%)

Alergias/intolerancias

Vegetarianismo

$$
89,8
$$

10,2

$21,0 \pm 2,99$

$3,08 \pm 1,44$

$4,95 \pm 1,74^{b}$

55,0

44,1

5,00

5,00

$$
81,8
$$

18,2

$20,6 \pm 2,49$

$3,39 \pm 2,32$

$6,24 \pm 1,78^{a}$

69,1

30,9

3,63

1,81
55,3

44,7

$20,4 \pm 2,62$

$2,84 \pm 1,11$

$5,27 \pm 1,40^{\mathrm{b}}$

69,4

29,2

2,04

0,00 
La comparación permitió apreciar una cantidad diaria de horas de estudio presenciales mayor en la carrera de BQ respecto de las dos Licenciaturas $(p<0,001)$; sin mostrar diferencias estadísticamente significativas en las demás variables estudiadas.

Actividad física $(A F)$ : El documento GAPA considera actividad física a cualquier movimiento corporal producido por los músculos esqueléticos que exija gasto de energía. El Mensaje 1 (M1) recomienda realizar al menos 30 minutos de AF diariamente; al respecto un $79,3 \%$ del total de estudiantes manifestó realizar AF, pero 59,8\% de ellos cumplía con el tiempo recomendado. Los tipos de AF más realizados fueron los de desplazamiento (danza, bicicleta/patín, caminata y/o trote/carrera), seguidos por los desarrolladas en gimnasios (pesas, crossfit), frecuentemente asociados a otra actividad encontrándose que el 39,2\% realizaba más de un tipo de AF. Solo un $8,5 \%$ utilizaba el predio deportivo que posee la UNL. La tabla 2 describe la AF por carreras y permite observar que $\mathrm{BQ}$ realizaba menos minutos semanales de $\mathrm{AF}$ que las Licenciaturas.

Adicionalmente, entre quienes admitieron realizar $\mathrm{AF}$, un $64,6 \%$ declaró haberla reducido al comenzar la universidad; mientras que, entre los que declararon no realizar $\mathrm{AF}$, un $54,5 \%$ las abandonó al comenzar los estudios.

Comidas diarias: El M1 aconseja realizar cuatro comidas diarias en un ambiente tranquilo y, en lo posible, acompañado. Se encontró que $\mathrm{LN}$ presentó mayor porcentaje de alumnos que realizan las 4 comidas recomendadas $(45,0 \%)$ respecto de LB $(22,4 \%)$ y BQ $(21,8 \%)(p=0,009)$. La figura 1 muestra el análisis de cada una de las comidas separadamente, observándose que el almuerzo fue la más realizada seguido por la cena, sin diferencias estadísticamente significativas entre carreras. La comida menos realizada para las tres carreras fue la merienda; entendiendo por tal, a alimentos que se ingieren a media tarde (entre el almuerzo y la cena). El desayuno y la merienda mostraron diferencias estadísticas significativas entre carreras ( $p=0,014$ y $p=0,008$, respectivamente), con un mayor cumplimiento por estudiantes de LN.
El análisis del tiempo dedicado a las comidas indicó que al menos el 51,6\% de los alumnos de LN cumplían con la recomendación; mostrando una diferencia estadísticamente significativa para el desayuno $(p=$ $0,043)$, almuerzo $(p=0,007)$ y merienda $(p=0,041)$ cuando se comparó con las otras carreras. La comida a la cual los alumnos de las tres carreras dedican más tiempo fue la cena.

Las comidas que los estudiantes realizaban en instalaciones de la facultad fueron almuerzo $(60,9 \%)$ y merienda $(36,6 \%)$. Los sitios seleccionados para almorzar, en orden de prioridad, fueron: $34,1 \%$

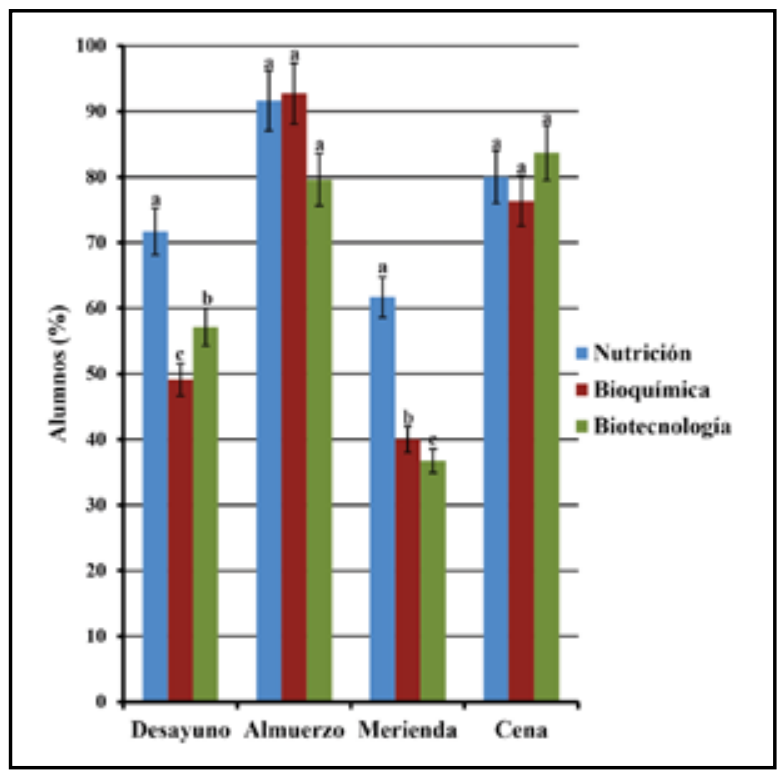

Figura 1: Porcentaje de alumnos por carreras que realizan diariamente cada comida principal. Los valores que no comparten letra superíndice son significativamente diferentes $(p<0,05)$.

Tabla 2. Actividad Física que realizan los alumnos discriminada por carreras. Los valores que no comparten superíndice son diferentes significativamente $(p<0.05)$.

\section{Actividad Física (AF)}

Realiza AF (\% de estudiantes)

Realiza $30 \mathrm{~min} /$ día (\% de estudiantes)

Tipo de actividad desarrollada (\%)

Natación
Deporte en equipos
Gimnasio

Actividades de desplazamiento

Tiempo semanal promedio (min)

\section{LN (n= 60)}

BQ (n= 55)

LB $(n=49)$

Total $(n=164)$

$\begin{array}{cccc}31,0 & 25,2 & 23,1 & 79,3 \\ 22,6 & 17,7 & 19,5 & 59.8 \\ & & & \\ 0,01 & 0,61 & 0,62 & 1,24 \\ 3,63 & 4,30 & 8,53 & 16,5 \\ 17,1 & 9,71 & 11,6 & 38,4 \\ 23,2 & 17,7 & 12,8 & 53,7 \\ 242 \pm 200^{\text {ab }} & 176 \pm 153^{\mathrm{b}} & 272 \pm 246^{\mathrm{a}} & \end{array}$


comedor universitario (perteneciente a la UNL), 28,6\% establecimientos privados (concesionados para el expendio de comidas y bebidas en la ciudad universitaria, que también cuentan con servicio de mesa), y $17,1 \%$ otras dependencias (parque, galerías de estudio). La merienda se realizaba en otras dependencias $(83,0 \%)$ y cantina $(38,9 \%)$. El desayuno y la cena eran realizados principalmente en sus hogares $(93,6 \%$ y $98,6 \%$, respectivamente). Respecto a la compañía, el desayuno fue la comida que más realizaban solos $(80,5 \%)$; las otras comidas refirieron hacerlas mayoritariamente acompañados: almuerzo $83,5 \%$, merienda $73,2 \%$ y cena $95,1 \%$.

Consumo de bebidas: Varias recomendaciones surgen en diferentes mensajes.

- Bebidas no alcohólicas: El M2 resalta la importancia de beber agua segura, al menos 8 vasos (2 litros) diariamente. Del total de alumnos $81,1 \%$ refirió consumir agua a diario, mientras $34,8 \%$ cumplió la cantidad recomendada.

EI M5 recomienda limitar el consumo de bebidas azucaradas y el agregado de azúcar a las infusiones. La figura 2 muestra el consumo de este tipo de bebidas discriminando entre versiones azucaradas y no azucaradas. En orden decreciente, el consumo de bebidas con azúcar fue: 55,5\% jugos y gaseosas; $41,5 \%$ infusiones (té, café, mate cocido) y $26,2 \%$ mate cebado. La variante mate cebado amargo fue consumida por $59,7 \%$ de los estudiantes. La mayor frecuencia de consumo de jugos y gaseosas azucaradas fue de 1 -2 veces/semana $(34,1 \%)$ y un $8,5 \%$ las consumía diariamente.

- Bebidas alcohólicas: El M10 indica su consumo responsable, no exceder 2 medidas de bebida alcohólica para varones y 1 para mujeres. El 17,1\% manifestó no beber alcohol. La cerveza y los aperitivos (fernet y vermú) fueron los más elegidos; $24,6 \%$ consumía uno a dos litros de cerveza, y $72,1 \%$ hasta un litro de aperitivos cada vez que bebían. En el $63,3 \%$ de los casos su consumo fue de 1-2 veces/semana.

\section{Consumo por grupos de alimentos}

- Carnes y huevo: El M7 recomienda consumirlos diariamente (hasta un huevo, especialmente en reemplazo de carne) y aumentar el consumo de pescado. Un 37,2\% consumía diariamente carnes y un 7,3\% huevo. En orden de preferencia las carnes seleccionadas fueron: pollo $(84,7 \%)$, vaca $(83,5 \%)$, pescado $(44,5 \%)$ y cerdo $(42,1 \%)$. Las formas de cocción más usadas para carnes fueron horno $(73,8 \%)$, plancha $(62,8 \%)$ y asada $(45,7 \%)$; y para huevo, hervido $(65,8 \%)$. La fritura fue la menos elegida $(15,2 \%$ en carnes y $8,5 \%$ en huevo).

- Lácteos: El M6 recomienda consumir diariamente leche, yogur o quesos (preferentemente blandos sobre duros), priorizando las versiones descremadas. El consumo diario de leche/yogur fue menor en BQ y LB respecto de $\mathrm{LN}(\mathrm{p}=0,042)$ y más del $70,0 \%$ optaba por las versiones descremadas, sin mostrar diferencia significativa entre carreras. La forma de consumo de leche más usual incluyó

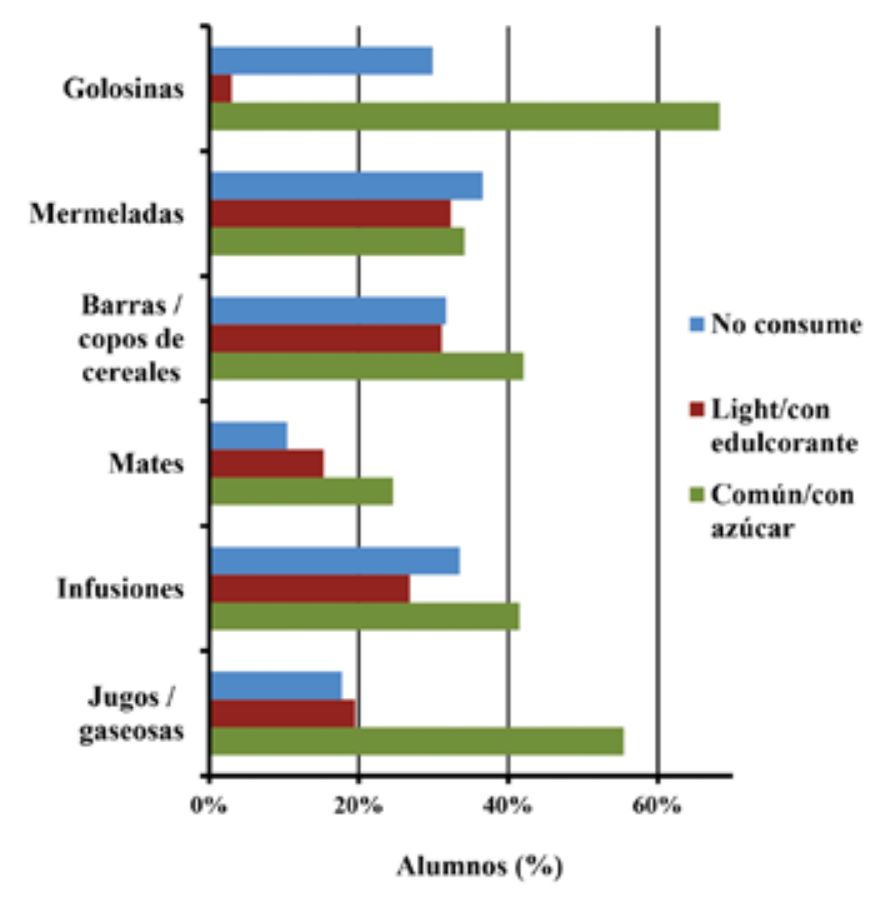

Figura 2: Preferencia en la selección de versiones con azúcar o light en alimentos y bebidas no alcohólicas. 
el agregado de azúcar y/o cacao (93,1\%). En relación a los quesos, el 51,2\% los consumía frecuentemente (5-7 veces/semana); la recomendación de elegir los blandos mostró una adherencia del $47,9 \%$ (variedades untable y/o cremoso), y de ellos 49,1\% seleccionaron descremados.

- Frutas y verduras: El M3 recomienda consumirlas a diario en variedad de tipos y colores. Se observó que la adherencia en estudiantes de LN duplica la de BQ y LB. Los estudiantes de BQ presentaron el consumo diario de frutas más bajo $(p<0,001)$, mientras los de $L B$ la menor frecuencia de ingesta de verduras diaria $(p=$ 0,023) (Figura 3).

- Feculentos cocidos y pan: Este grupo incluye los cereales y sus derivados (como las pastas), las legumbres, los vegetales feculentos (papa, batata, choclo y mandioca) y el pan. El M8 recomienda el aumento del consumo de legumbres y cereales integrales.

La elección de las diferentes variedades de este grupo fue: $96,9 \%$ cereales y derivados, $64,6 \%$ legumbres, $82,9 \%$ vegetales feculentos y $80,5 \%$ pan.

La figura 4 muestra la frecuencia de consumo de este grupo; no se encontró diferencia estadísticamente significativa entre carreras. La mayor frecuencia de consumo de cereales y derivados fue $3-4$ veces/semana $(40,9 \%)$; adicionalmente, un $17,9 \%$ los consumía en sus variedades integrales. Los vegetales feculentos fueron consumidos con mayor frecuencia $1-2$ veces/semana $(36,8 \%)$. Las legumbres también se seleccionaron con mayor frecuencia $1-2$ veces/semana ( $41,7 \%)$; sin embargo, fueron las menos consumidas del grupo: $35,0 \%$ manifestó no consumirlas.

El consumo de pan presentó el siguiente patrón: la mayor frecuencia $(27,6 \%)$ fue de $1-2$ veces/semana, $25,2 \%$ diariamente, $19,6 \%$ de los estudiantes no lo consumía.

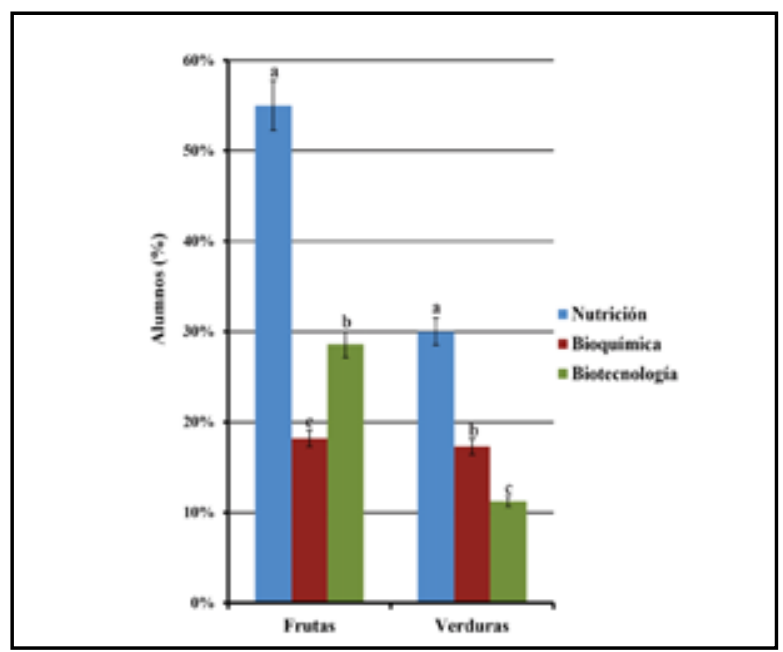

Figura 3: Porcentaje de alumnos por carreras que consumen diariamente frutas y verduras. Los valores que no comparten letra superíndice son significativamente diferentes $(\mathrm{p}<0.05)$.
Una práctica semejante se verificó para galletas, alimento que las GAPA recomienda consumir como reemplazo del pan: $23,7 \%$ lo seleccionaba $1-2$ veces/semana, $18,9 \%$ lo hacía diariamente y $26,2 \%$ no lo consumía. La selección de variedades integrales fue $30,5 \%$ para pan y $42,1 \%$ para galletas.

- Frutos secos y semillas: El M9 recomienda su consumo sin agregado de sal, al menos una vez a la semana. Se halló que $43,9 \%$ cumplía esta recomendación para los frutos secos y $37,8 \%$ para las semillas. Un $43,9 \%$ nunca los consumía.

- Grupo opcional (dulces y grasas): El M5 menciona limitar el consumo de alimentos ricos en azúcares simples, grasas y sal. Ampliando dicho mensaje (submensaje 1) se señala limitar el consumo de amasados de pastelería y productos de copetín (como palitos salados, papas fritas de paquete, etc.). Los resultados mostraron que el $72,6 \%$ consumía este tipo de pastelería y el 51,8\% productos de copetín. En ambos casos, el 3,1\% los consumía frecuentemente (5-7 veces a la semana).

La figura 2 muestra la selección de versiones comunes o light discriminada por alimentos. Para las barras y copos de cereales, mermeladas y golosinas prima la selección de las versiones con azúcar. El consumo frecuente fue: barras/copos de cereales 20,1\%, mermeladas $34,7 \%$ y golosinas del 10,9\%.

- Sal y alimentos ricos en sodio: El M4 recomienda reducir el uso de sal, como también el consumo de fiambres, embutidos y productos industrializados. El 18,9\% de los estudiantes agregaba sal a las preparaciones servidas ya saladas. La mayor frecuencia de consumo de fiambres y embutidos, fue de 1-2 veces/semana en el $51,2 \%$ de los estudiantes.

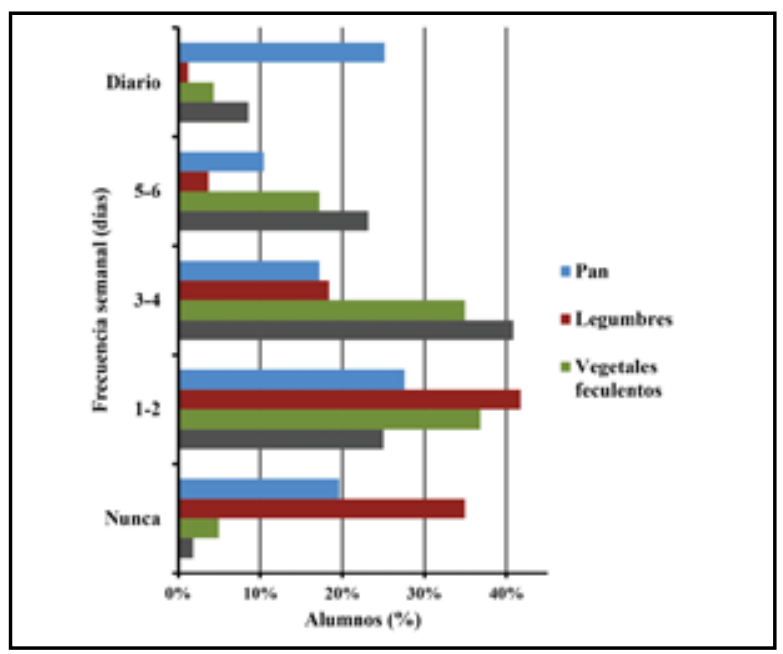

Figura 4: Frecuencia de consumo del grupo feculentos cocidos y pan. 


\section{DISCUSIÓN}

En Argentina, según la $3^{\circ} \mathrm{ENFR}^{15}$, las personas con mayor nivel educativo presentaron mejor estilo de vida que aquellas con un nivel de instrucción menor. A la luz de estos resultados cabría esperar en los estudiantes universitarios estilos de vida cercanos a las recomendaciones propuestas desde el Ministerio de Salud. Aunque existen trabajos previos realizados sobre estilos de vida en universitarios argentinos, al presente no se cuenta con información suficiente que estime su adecuación a las nuevas GAPA. Esta investigación permite conocer aspectos contextualizados acerca de su alimentación y actividad física.

La caracterización de la muestra universitaria en su contexto contribuyó al análisis crítico en la valoración y comparación de la alimentación y AF de los estudiantes, objetivo de la presente investigación. Como hallazgo se reveló que los estudiantes contaban con un año más de vida universitaria que el esperado de acuerdo al programa de estudio de cada carrera; ese desfase podría resultar indicativo del proceso de adaptación que esta etapa requiere. Una cantidad considerable pertenecía a la ciudad donde estudia, cuestión que suele asociarse a la posibilidad de contención en el seno familiar; sin embargo, una carga importante de horas de cursado los obligaba a permanecer en el campus universitario en largas jornadas. Esto último podría condicionar las selecciones y patrones alimentarios y las posibilidades de realizar $\mathrm{AF}^{16}$.

Científicamente, se reconoce la importancia de realizar AF a la par de una alimentación saludable en la prevención de $\mathrm{ECNT}^{17}$. Un aspecto destacable de las GAPA 2016 en comparación con sus predecesoras (GAPA 2002) es la incorporación explicita de realizar AF en forma diaria en su primer mensaje y en la gráfica que las acompaña. La mayoría de los estudiantes $(79,3 \%)$ realizaba algún tipo de actividad, resultado coincidente $(79,8 \%)$ con el de otro estudio en universitarios argentinos de La Matanza (Bs As) de diferentes carreras (sociales y exactas) ${ }^{18}$. Además, en el mencionado trabajo se indica que la $\mathrm{AF}$ se vio favorecida -entre otros factores- por el cursado diurno, condición semejante a la de los alumnos del presente trabajo.

Como aporte de esta investigación se destacan a continuación aspectos sobre la AF que resultan importantes. La cantidad de alumnos es menor (59,8\%) si se considera quienes cumplen el tiempo recomendado por GAPA, en este sentido el ingreso a la universidad implicó abandono o reducción del tiempo dedicado, y el uso del predio deportivo de la universidad fue bajo. En estudiantes de UNL, otro trabajo encontró que 50,8\% cumplía las recomendaciones de OMS (150 minutos semanales de AF moderada) ${ }^{4}$; en el presente trabajo, las tres carreras alcanzaron dicho promedio, aunque las desviaciones estadísticas resultaron muy elevadas indicando una gran variabilidad. Si bien el presente trabajo no investigó los motivos asociados, la disminución de la AF motivada por cambios de patrones conductuales hacia estilos de vida sedentarios en las sociedades modernas es un factor a tener en cuenta ${ }^{19,20}$. Más aún, se demostró la problemática emergente que genera el consumo de videojuegos (frecuencia de juego y cantidad) sobre los hábitos saludables en la etapa universitaria ${ }^{21}$. La falta de tiempo aparece frecuentemente como un factor importante en los individuos que no realizan AF y así quedó demostrado en la población argentina ${ }^{15}$. La influencia de este factor se registró en estudiantes chilenos ${ }^{22}$ y en otros universitarios argentinos donde además se menciona el hecho que la mayor actividad física se registra en el tiempo libre $^{18}$. En el presente trabajo se constató que los estudiantes de las tres carreras investigadas en la FBCB tienen una cantidad de horas de estudio diario que podría disminuir el tiempo libre dedicado a AF; lo cual también podría ser un factor vinculado a la poca utilización del predio de deportes de la universidad, aun cuando éste se halla ubicado lindante a la Facultad.

En relación a los patrones alimentarios y las GAPA, los estudiantes de $\mathrm{LN}$ mostraron mejor adopción de las recomendaciones que sus pares de $B$ y $L B$ en varios aspectos. Otro trabajo de nuestro grupo ${ }^{23}$, también realizado en el proyecto marco, evaluó conocimientos sobre alimentación saludable y los mensajes de las GAPA en 526 alumnos del ciclo básico $\left(1^{\circ}\right.$ a $3^{\circ}$ año) de las tres carreras de FBCB-UNL y encontró que el conocimiento del documento GAPA resultó significativamente mayor para $\mathrm{LN}: 84,6 \% \mathrm{LN}, 8,51 \%$ B y $6,9 \%$ LB $(p<0,0001)$. Aunque en este trabajo la encuesta no se diseñó para relacionar conocimiento con prácticas, el momento de aplicación de la misma $\left(2^{\circ}\right.$ año) determina que un mayor conocimiento de las GAPA existiría sólo para aquellos estudiantes de $\mathrm{LN}$ que al momento de responderla estuviesen cursando su primera asignatura sobre fundamentos de alimentación y nutrición. Sin embargo, la motivación inherente a la carrera elegida (LN) podría actuar como un factor que no debería ser soslayado. Investigaciones sobre patrones y/o hábitos alimentarios comparativos entre carreras difieren según las universidades y no existe uniformidad en las consideraciones basadas en los conocimientos que poseen sus alumnos. Así, un grupo de trabajos no encontraron diferencias entre titulaciones y refieren que los conocimientos no influyeron en la toma de decisiones para una alimentación y estilo de vida saludables ${ }^{6,24,25}$; mientras otros atribuyen ventajas a los universitarios con mayor conocimiento ${ }^{21,26,27,28}$.

Actualmente se reconoce que el número de ingestas diarias es importante para considerar una dieta como saludable $^{29}$. La recomendación de GAPA (realizar cuatro comidas diarias) mostró un cumplimiento significativamente mayor en estudiantes de LN, pero aun así no alcanzó a la mitad (45\%). Dicho valor es semejante al encontrado en otros universitarios de nuestro país (44\%), donde además se observó que el número inadecuado de ingestas reportaba el doble de riesgo de padecer grasa corporal incrementada ${ }^{29}$.

Un aspecto a tener en cuenta si se piensa en términos de promoción de una universidad saludable, es que el almuerzo resultó la comida realizada mayoritariamente en la Facultad por los estudiantes de las tres carreras. La UNL cuenta con 
comedor propio que ofrece un menú nutricionalmente adecuado a un costo razonable en el predio universitario; no obstante, esta opción -que podría ser considerada una ventaja- resultó seleccionada por algo más de un tercio de los estudiantes. Este dato revela la necesidad de profundizar la investigación de los motivos y el abordaje de las causas subyacentes, a fin de incrementar la utilización de los espacios saludables que la universidad ofrece.

Con el fin de orientar buenas prácticas nutricionales resulta útil el término "brechas alimentarias", el cual ha sido empleado por Britos et a ${ }^{30}$ para valorar lo que comen los argentinos en relación a lo que deberían comer en términos de una alimentación saludable; estimando estas brechas como la diferencia entre las cantidades consumidas de diferentes grupos o categorías de alimentos respecto de las recomendadas por distintas guías alimentarias. Si bien el presente trabajo presenta como limitación que no se estimaron las cantidades ingeridas, bajo un paradigma semejante se procuraron establecer "brechas alimentarias" en relación a hábitos y frecuencias de consumo para los diferentes grupos de alimentos tomando como parámetro las recomendaciones de las nuevas GAPA acorde a los objetivos planteados.

Las recomendaciones de consumo diario para carnes, un alimento que forma parte de la cultura alimentaria y es de alta ponderación en el patrón alimentario de los argentinos, se cumple en algo más del tercio de los estudiantes; siendo su forma de cocción mayoritariamente saludable (al horno, plancha o asada). Puesto que existe una elección mayoritaria de carne de vaca y pollo, en consonancia con la población general, es necesario resaltar -como recomienda el M7la necesidad de incrementar el consumo de pescado. Particularmente beneficioso sería el consumo de pescados de mar ricos en ácidos grasos n-3, más aún si se tiene en consideración que otras fuentes como semillas y frutos secos resultaron poco consumidas por estos estudiantes. Estos patrones de alimentación resultan fuertemente asociados a la cultura; en Colombia se encontró que los universitarios consumían preferentemente pollo (39\%), seguido de pescado $(37 \%)^{31}$. El consumo de pescado junto al de frutos secos y aceite de oliva, característicos de la dieta mediterránea y considerados eficaces en la prevención y el desarrollo de ECNT, también han mostrado en los últimos años una menor adherencia a las recomendaciones en la dieta de los universitarios ${ }^{18,31,32}$.

En este estudio quedó demostrado que el consumo de frutas y verduras es bajo, aunque los estudiantes de LN presentaron una mejor adecuación a las recomendaciones. En Argentina, la $3^{\circ}$ ENFR puso en evidencia un patrón de escaso consumo (promedio de 1,9 porciones/día), lejos de la meta de 5 porciones diarias de las $\mathrm{GAPA}^{15}$; sin mostrar cambios en la $4^{\circ}$ ENFR $^{33}$ donde solo el $6 \%$ de la población cumplió la recomendación. Este patrón también se encontró en estudios latinoamericanos e internacionales ${ }^{10}$, aunque en universitarios de Alicante (España) el consumo de frutas y verduras mejoró un $100 \%$ de un año a otro en estudiantes de ciencias de la salud ${ }^{28}$. Otras fuentes de fibra alimentaria como los cereales integrales y las legumbres -adicionalmente propuestos en el M8 como sustitutos para la carne- mostraron un escaso consumo. Este comportamiento presente en la cultura argentina, también fue observado en otros universitarios del país ${ }^{34}$, incluido un estudio previo de nuestro grupo en estudiantes de medicina de la UNL ${ }^{5}$. En otras culturas, los estudiantes tampoco llegan a cubrir sus recomendaciones ${ }^{35,36}$. A pesar que algunos investigadores españoles refieren una pérdida de adherencia a la dieta mediterránea en sus estudiantes universitarios, comparativamente presentan un mejor patrón de consumo de legumbres que los de Argentina.

Especial atención requiere el consumo de los nutrientes que tanto las GAPA como la OMS indican limitar, ya que su exceso resulta perjudicial en relación a las ECNT: azúcares simples, grasa y sal. El agregado de sal a los alimentos ya servidos (30\%) coincide con un trabajo previo ${ }^{5}$; sin embargo, resulta superior a lo hallado en las ENFR, donde además se verificó una disminución del 25,3\% registrado en 2009 al $16,4 \%$ en $2018^{33}$. Los dulces y golosinas, junto a los amasados de pastelería (ricos en azúcar y grasa), resultaron consumidos ampliamente por los estudiantes. Esta selección alimentaria se realizaba en mayor medida como colación entre comidas o merienda en la Facultad por su practicidad, disponibilidad y precio, lo que evidentemente compite y gana terreno respecto a aquellos alimentos más saludables.

Investigaciones en universitarios señalan que la tendencia de consumo de alimentos azucarados parece ir en aumento ${ }^{34,37}$. Las golosinas resultaron los productos más consumidos entre los alimentos azucarados, en coincidencia con otro estudio donde las mismas aparecieron como primera elección en la cantina universitaria ${ }^{38}$. Las versiones light o con edulcorante, aunque inferiores a las azucaradas, lograron una mayor adherencia en otros alimentos como barra/copos de cereales y mermeladas.

El consumo de bebidas azucaradas también fue preponderante y los jugos/gaseosas resultaron seleccionados en mayor medida. La frecuencia de consumo diario (8\%) resultó inferior la observada en estudiantes colombianos $(17 \%)^{22}$ y españoles $(19 \%)^{39}$. Adicionalmente, la selección de versiones light $(20 \%)$ resultó superior a la observada en estudiantes universitarios de Chile $(14 \%)^{40}$. Enfocándose en la modificación del comportamiento alimentario, han sido reportadas diversas estrategias para la disminución del consumo de bebidas endulzadas ${ }^{37}$. En Argentina, las GAPA (pág. 69) reconocen que estas bebidas lideradas por las gaseosas comunes están creciendo como consecuencia de factores macroeconómicos que impulsaron su consumo; al presente, no se han elaborado estrategias específicas para disminuir su consumo.

La Universidad ofrece espacios de alimentación saludable (comedor universitario) y actividad física (campo de deportes), posibilitadores de estilos de vida saludables que deben ser mejor aprovechados. Estos espacios saludables, junto a intervenciones áulicas apropiadas y sistemas de difusión 
adecuados, son importantes herramientas para potenciar acciones concretas. Es innegable que la educación alimentaria nutricional es muy necesaria, por ello se debe trabajar de igual modo en todas las carreras. Esto significaría empoderar a todos los alumnos de saberes que, además, pueden ser retransmitidos a sus familias, pares y la sociedad en general.

\section{CONCLUSIONES}

En la FBCB, los estudiantes de LN mostraron un mejor cumplimiento de las recomendaciones para una alimentación saludable que los de BQ y LB. No obstante, se pudo observar que las tres carreras presentan patrones de consumo similares a la población argentina general que deben ser mejorados, alejándose principalmente de las recomendaciones respecto al consumo de frutas, verduras, cereales integrales y legumbres, y con un consumo importante de nutrientes cuyo exceso resulta perjudicial en el desarrollo de ECNT. La proporción de estudiantes que realizaba actividad física no resultó baja, sin embargo, debe promoverse un incremento en la cantidad de tiempo dedicado a la misma para que un mayor número alcance las recomendaciones.

Esta investigación permite conocer aspectos contextualizados acerca de la alimentación y actividad física de estudiantes de tres carreras, necesarios para pensar y ejecutar acciones de promoción en salud como lo proponen los lineamientos para las universidades saludables en nuestro país y en el mundo. Del mismo modo, brinda un punto de partida necesario para aceptar el reto que se impone a los círculos académicos en la lucha contra las ECNT a través de las indicaciones de las GAPA 2016 y el reciente informe de OMS.

Agradecimientos: Esta investigación fue financiada por el Proyecto CAI+D 2016-UNL PI 50120150100042LI.

\section{BIBLIOGRAFÍA}

1. World Health Organization. Global strategy on diet, pyshical activity and health, Ginebra 2004.

2. World Health Organization. Time to deliver: report of the WHO Independent high-level commission on noncommunicable diseases, Ginebra 2018.

3. Dirección de Estadísticas e Información de Salud (DEIS - MSAL). Birth rate and mortality 2016. Statistical Synthesis 3, Buenos Aires 2018.

4. Negro E, Gerstner C, Depetris C, Barfuss A, González M, Williner MR. Prevalence of cardiovascular disease risk factors in university students in Santa Fe (Argentina). Rev Esp Nutr Hum Diet. 2018; 22: 132-140.

5. Monsted C, Lazzarino MS, Modini LB, Zubriggen A, Fortino MA. Anthropometric evaluation, dietary intake and level of physical activity in medical students of Santa Fe (Argentina). Rev Esp Nutr Hum Diet. 2014; 18: 3-11.

6. Cedillo Ramirez L, Correa-López $L E$, Vela-Ruiz JM, PerezAcuña LM, Loayza-Castro JA, Cabello-Vela CS, et al. Styles of life of university students of health sciences. Rev Fac Med Hum. 2016; 16: 57-65.
7. World Health Organization. The Ottawa Charter for Health Promotion, Ottawa 1986.

8. Navarro-Prado S, Gonzáles-Jiménez E, Montero-Alonso MA, López-Bueno M, Schmidt-Rio Valle J. Lifestyle and monitoring of dietary intake in students of University of Granada Campus in Melilla. Nutr Hosp. 2015; 31: 26512659.

9. Becerra S. Description of health behaviors in a group of university students from Lima. Rev Psicol. 2016; 34: 239260.

10. Sánchez-Ojeda MA, De Luna-Bertos E. Healthy life habits in the university population. Nutr Hosp. 2015; 31: 19101919.

11. Ministerio de Salud de la Nación. Manual of Healthy Universities, Buenos Aires 2012.

12. Ministerio de Salud de la Nación. Dietary Guidelines for the Argentine Population, Buenos Aires 2016.

13. Walpole RE, Myers RH, Myers SL, Ye K. Statistics and probability for science and engineering. 9 th ed. México: Pearson Educación, 2012.

14. Menal-Puey S, Fajó-Pascual M, Marques-Lopes I. Descriptive study of breakfast in a school-based immigrant child population, 2007-2010. Rev Esp Nutr Hum Diet. 2011; 15: $177-183$.

15. Ministerio de Salud de la Nación. Third National Survey of Risk Factors for Non-Communicable Diseases, Buenos Aires 2015.

16. Duarte-Cuervo CY, Ramos-Caballero DM, Latorre-Guapo AC, González-Robayo PN. Factors related to students' eating practices in three universities in Bogotá, Colombia. Rev Salud Pública. 2015; 17: 925-937.

17. Ramírez-Vélez R. Physical activity and quality of life related to health: systemic review of current evidence, Rev Andal Med Deporte. 2010; 3: 110-120.

18. Pérez Ugidos G, Laíño FA, Zelarayán J, Márquez S. Physical activity and health habits in Argentine university students. Nutr Hosp. 2014; 30: 896-904.

19. Montero Bravo A, Úbeda Martín N, García González A. Evaluation of the dietary habits of a population of university students in relation to their nutritional knowledge, Rev Nutr Hosp. 2006; 21: 466-473.

20. Chales-Aoun AG, Escobar JMM. Physical activity and eating behaviors among chilean university students. Cienc Enferm. 2019; 25: 1-10.

21. Chacón-Cuberos $R$, Castros-Sánchez M, Muros-Molina J, Espejo-Gracés T, Zurita-Ortega F, Linares-Manrique $M$. Adherence to the Mediterranean diet in university students and their relationship with digital leisure habits. Nutr Hosp. 2016; 33: 405-410.

22. Díaz-Muñoz GA. Characterizing eating habits and physical activity levels in college students. Rev Salud Bosque. 2018; 8: 9-19.

23. Dezar GVA, Vargas MR, Ravelli SD, Cuneo F, Ávila OB, Fortino MA. Knowledge and practice of recommendations on healthy food in university students. Actual Nutr. 2019; 20: 105-111.

24. Muñoz de Mier G, Lozano Estevan MC, Romero Magdalena CS, Pérez de Diego J, Veiga Herreros P. University students' food consumption assessment and the relation with their academic profile. Nutr Hosp. 2017; 34: 134-143.

25. San Román MS, Ortega FZ, Martínez AM, Ruz RP, Cuberos $R C$, Manrique ML. Adherence of the mediterranean diet in university students in southern Spain according to social, 
academic and religious factors. Rev Esp Nutr Hum Diet. 2018; 22: 141-148.

26. Vega MR, Manzanera JM, González Panero MP, Gurruchaga MT. Changes in the adherence to the mediterranean diet in students of nursing and teaching degrees after taking a nutrition course Nutr Hosp. 2014; 30: 1173-1180.

27. Kolodinky J, Harveu-Berino JR, Berlin L, Johnson RK, Reynolds TW. Knowledge of current dietary guidelines and food choice by college students: Better eaters have higher knowledge of dietary guidance I Am Diet Assoc. 2007; 107: 1409-1413.

28. Sánchez-Fideli MA, Gutiérrez-Hervás A, Rizo-Baeza $M$, Cortés-Castell E. Longitudinal study on adherence to the Mediterranean diet pattern in students of health sciences. Rev Esp Nutr Hum Diet. 2018; 22: 4-13.

29. Pi RA, Vidal PD, Brassesco BR, Viola $L$, Aballay $L R$. Nutritional status in university students: its relationship with the number of daily food intakes and the consumption of macronutrients. Nutr Hosp. 2015; 31: 1748-1756.

30. Britos S, Saraví A, Vilella F. Good practices for a healthy diet for Argentine population, Universidad de Buenos Aires $1^{\circ}$ ed., Buenos Aires, 2010.

31. Rodríguez $H$, Restrepo $L F$, Urango LA. Characterization of the consumption of meat products in a university population of the city of Medellín, Colombia. Rev Esp Nutr Hum Diet. 2015; 19: 90-96.

32. Pastor R., Bibilon MM, Tur Mari JA. Patterns of food consumption in Zamora university students. Nutr Hosp. 2017; 34: 1424-1431.
33. Ministerio de Salud y Desarrollo Social. $4^{\circ}$ National Survey of Risk Factors for Non-Communicable Diseases. Buenos Aires 2019.

34. De Piero A, Bassett N, Rossi A, Sammán N. Trends in food consumption of university students. Nutr Hosp. 2015; 31: 1842-1831.

35. Redondo del Río MP, Silleras BM, Enciso LC, de Mighuelsanz JMM, MCPhee MF, Martín MAC. Dietary intake and adherence to the Mediterranean diet in a group of university students based on sports practice. Nutr Hops. 2016; 33: 1172-1178.

36. Sánches Socarrás V, Aguilar Martínez A. Eating habits and behaviors related to health in a university population. Nutr Hosp. 2015; 31: 449-457.

37. García Flores CM, López-Espinoza A, Martínez Moreno AG, Miranda CP, Zepeda-Salvador AP. Strategies for reducing the consumption of sugary drinks. Rev Esp Nutr Hum Diet. 2018; 22: 169-179.

38. Hilger J, Loerbroks A, Diehl K. Eating behaviour of university students in Germany: Dietary intake, barriers to healthy eating and changes in eating behaviour since the time of matriculation. Appetite. 2017; 109: 100-107.

39. Zaragoza-Martí A, Norte-Navarro A, Fernández-Sáez J, Hurtado-Sánchez JA, Ortiz-Moncada R. Type of beverages consumed by university students. Rev Esp Nutr Comunitaria 2013; 19: 114-119.

40. Rodríguez F, Palma X, Romo A, Escobar D, Aragú B, Espinoza $L$, et al. Eating habits, physical activity and socioeconomic status in university students in Chile. Nutr Hosp. 2013; 28 : 447-455. 